\title{
Why doesn't sleep advice talk about sex?
}

\author{
Cite as: CMAJ 2021 March 15;193:E384-5. doi: 10.1503/cmaj.1095926
}

Posted on cmajnews.com on February 25, 2021

H alf of all Canadians report that they have trouble falling asleep and staying asleep at the best of times. The stress of living through a pandemic is only making matters worse. Yet advice on insomnia seldom mentions a potential nonpharmacological sleep aid that's free and easily accessible to most people: sex, whether with a partner or alone.

After orgasm, levels of oxytocin and prolactin rise while levels of cortisol drop. Common sense suggests that raising the level of feel-good hormones in the body and reducing stress hormone levels is likely to help us sleep better. Yet data on sex and sleep are scarce, due partly to taboos and squeamishness.

Dr. Judith Davidson, a Queen's University clinical psychologist and sleep researcher, was surprised by the dearth of good quality research on sex and sleep when she wrote the second edition of her insomnia self-help book, Sink into Sleep. "I really don't know why evidence is lacking," she says. "Perhaps the researchers in the sleep field and those in the sex field have not looked much beyond their respective academic borders. It has puzzled me."

So far, only one small study of 10 people has examined sleep after sexual activity using polysomnography - the gold standard test for diagnosing sleep disorders, which measures brain waves, heart rate and other indicators. Published in 1985, the study concluded that masturbation had no significant impact on sleep.

But the study had other limitations beyond its small size. The researchers may have interrupted participants' sleepiness after masturbation by removing an anal temperature probe. What's more, the study compared sleep after masturbation and sleep after reading - a known sleep inducer - which may have blunted differences between intervention and control.

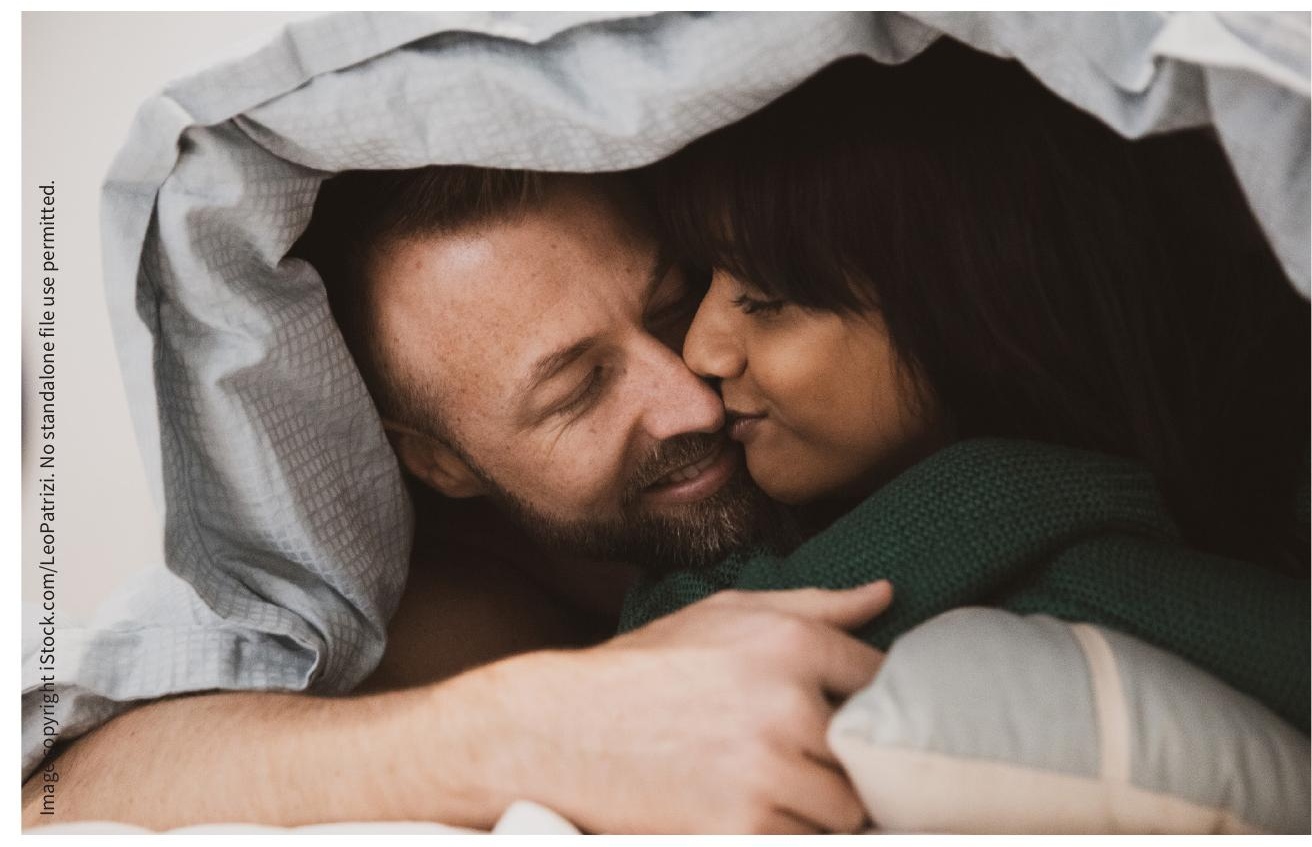

A big $\mathrm{O}$ may help people catch Zs, but research and guidelines have tended to overlook sex as a potential sleep aid.

More recent research suggests that sex can reduce stress and help people with insomnia fall asleep and stay asleep. Although the evidence base is small and some studies lack objective measures, a 2016 review by University of Ottawa researchers concluded that sex is a "possible alternative or addition to other intervention strategies for insomnia."

It's a conclusion that many people seem to reach by themselves. A 2019 study of 778 people led by Dr. Michele Lastella of the Central Queensland University in Australia found that both men and women perceive that they fall asleep quicker and sleep better after orgasm with a partner or alone.

According to Lastella, the link is worth exploring given that many people have trouble sticking to the usual sleep advice. Setting a bedtime, exercising regularly, and avoiding screens all require discipline and long-term commitment to pay off. He says switching "screen time for play time" may be an easier sell with more immediate results.

\section{Overcoming barriers}

Getting such advice into clinical guidelines is another matter. According to Dr. Charles Samuels, past president of the Canadian Sleep Society, "with so many taboos related to sex, opening that door is overwhelming."

Asked why sleep advice seldom mentions sex, Samuels cites clinical inertia given the other treatment options available and a lack of rigorous research.

"Sleep researchers pride themselves on high-quality, objective markers and sex research is difficult," Samuels says. However, he acknowledges that the next generation of clinicians and researchers may be more interested in tackling the topic. 
According to Lastella, ethical issues often hamper studies that involve sex, video, and researchers entering a room where sex takes place. There are also logistical challenges, such as drawing blood samples to measure hormonal activity during and after orgasm without interrupting sleepiness.

Expensive portable polysomnography units could help address some of these issues by bringing the lab to the bedroom rather than the bedroom to the lab. But Lastella says that reducing the stigma surrounding sex and sleep remains a far bigger obstacle to overcome.

Dr. Caroline Pukall, director of the Sexual Research Lab at Queen's University, says that many of her patients already use masturbation as a sleep aid. "Patients often cite that it is part of their bedtime routine... and that it's something that helps them to relax and fall asleep."
But while there are multiple sources of funding for sex research in Canada, Pukall says sex researchers still face an uphill battle to legitimize their field and secure ethics approval for studies. She has started giving educational talks to ethics review boards, which have often regarded sex research as a "frivolity... nice to know but not need to know."

Dr. Stephanie Hart, a family physician with a focus on sexual medicine in Okotoks, Alberta, says such talks should start in medical school and residency.

She says that discussions about the importance of healthy sexuality notably lack in medical training. Instead, "the focus is on sexually transmitted infections and dysfunction." Within that framing, "sex is bad, dirty, even gross, so we don't talk about that. We don't bring it up because we are embarrassed, and then patients are embarrassed."
But a more sex-positive perspective is gaining traction. Hart has received multiple invitations to talk about sexuality at the University of Calgary, student groups and medical conferences. "I tell them if they can practise saying words like anal sex, masturbation and clitoris over and over until they stop blushing, then maybe we can have some hope that patients will be able to discuss this important part of their health."

Dr. Yuliya Rackal, Toronto, Ont.

Content licence: This is an Open Access article distributed in accordance with the terms of the Creative Commons Attribution (CC BY-NC-ND 4.0) licence, which permits use, distribution and reproduction in any medium, provided that the original publication is properly cited, the use is noncommercial (i.e., research or educational use), and no modifications or adaptations are made. See: https://creativecommons.org/ licenses/by-nc-nd/4.0/ 